\title{
In situ Liquid Cell Transmission Electron Microscopy Study of Hydroxyapatite Mineralization Process
}

\author{
Kun $\mathrm{He}^{1}$, Cong Liu ${ }^{2}$, Yifei Yuan ${ }^{2,3}$, Boao Song ${ }^{3}$, Yu-peng Lu*, Tolou Shokuhfar ${ }^{3,5 *}$, Reza Shahbazian- \\ $\operatorname{Yassar}^{3 *}$ \\ 1. The NUANCE Center, Northwestern University, Evanston, IL, USA. \\ 2. Chemical Sciences and Engineering Division, Argonne National Laboratory, Argonne, IL, USA \\ 3. Department of Mechanical and Industrial Engineering, University of Illinois at Chicago, Chicago, IL, \\ USA \\ 4. School of Materials Science and Engineering, Shandong University, Ji'nan, China. \\ 5. Department of Bioengineering, University of Illinois at Chicago, Chicago, IL, USA. \\ *Corresponding author: rsyassar@uic.edu
}

Human hard tissues are composed of collagen microfibril and bioapatite crystals, also called hydroxyapatite (HA) [1]. As an example, the teeth enamel has the highest concentration ( $\sim 96 \mathrm{wt} \%$ ) of HA minerals [2], and bone tissue is made of $70 \mathrm{wt} . \%$ mineral (with nano-sized HA being the majority phase [3]. The mineralization process of HA is essentially controlled by the nucleation and growth of crystalline HA at the microscopic level. Dysfunctional or aberrant mineralization leads to a variety of medical problems, such as decays in teeth and osteoporosis that are related to the demineralization of HA, and arteriosclerosis that is related to the unwanted mineralization of HA. The biomineralization of HA is the core reaction determining the health conditions of hard tissue. The repair of bone and hard tissue or overcoming pathological calcification in soft tissue requires the detailed understanding of biomineralization pathways of calcium phosphate materials. It is generally accepted that during the mineralization process of HA, amorphous calcium phosphate (ACP) plays an important role. However, the details regarding how ACP transforms to HA are still unclear. While it is accepted that ACP as the intermediate phase can provide energetically favorable landscape for HA formation, it is not clear how they transform to HA crystals. Here, we report the first in-situ liquid-cell transmission electron microscopy (TEM) study of HA mineralization process in an artificial saliva solution. We demonstrate the coexistence of the classical and non-classical nucleation in the HA mineralization process. It is found that the mineralization of HA starts with a liquid-liquid phase mechanism separating the saliva media into ionrich and ion-poor solutions. The direct evidence could be recorded by in-situ liquid cell TEM. Interestingly, we observed that in addition to the formation of ACP nanoparticles, smaller crystals of HA also formed simultaneously within the ion-rich solution. While ACP nanoparticles can grow in size up to $150 \mathrm{~nm}$, the HA crystals do not grow and maintain sizes below $10 \mathrm{~nm}$. The transition of ACP to HA starts by the heterogeneous nucleation of HA crystals on ACP nanoparticles at the solution-ACP interfaces followed by the dissolution of ACP. The growth of HA crystals is governed by the aggregation mechanism. This new understanding is important to address the physiological and pathological formation of HA minerals, such as improve the biomineralization to help the repair of hard tissue or stop the growth of HA to prevent the pathological calcification at nanoscale.

References:

[1] J. D. Pasteris, B. Wopenka, E. Valsami-Jones, Elements 4 (2008), p. 97.

[2] Y.-R. Zhang et al., Int. J. Oral Sci. 6 (2014), p. 61.

[3] L. C. Palmer et al., Chem. Rev. 108 (2008), p. 4754. 
https://doi.org/10.1017/S1431927619008249 Published online by Cambridge University Press 